\title{
Polypharmacy: A Risk Factor for Geriatric Syndrome, Morbidity \& Mortality
}

\section{Abdulraheem IS*}

Associate Professor, University of Ilorin, Nigeria

The number of elderly is increasing worldwide and among them, polypharmacy is a reoccurring issue. Polypharmacy is simply defined as the use of multiple medications by a patient. This definition excludes topical and herbal medications as they are often not included in the traditional methods of assessing prescription quality. Vitamins and minerals taken as much needed by individual are also generally excluded in because of the inconsistent inclusion of these medications in polypharmacy. The precise minimum number of medications used to define "polypharmacy" is variable, but generally ranges from 5 to 10 [1]. While polypharmacy most commonly refers to prescribed medications, it is important to also consider the number of over-the-counter and herbal drugs or supplements used by the elderly. Polypharmacy is an important issue in older people who, compared to younger individuals, tend to have more disease conditions for which therapies are prescribed. It has been estimated that 20 percent of Medicare beneficiaries have five or more chronic conditions and 50 percent receive five or more medications [2]. Older adults are seriously focused regarding polyphamacy because of the following reasons: (1) Older individuals are at greater risk for adverse drug events due to metabolic changes and decreased drug clearance associated with ageing; this risk is compounded by increasing numbers of drugs used. (2) Polypharmacy increases the potential for drug-drug interactions. (3). Polypharmacy was an independent risk factor for hip fractures in older adults in one case-control study, although the number of drugs may have been an indicator of higher likelihood of exposure to specific types of drugs associated with falls (eg, CNS active drugs) [3]. (4) Polypharmacy increases the possibility of "prescribing cascades" [4]. A prescribing cascade develops when an adverse drug event is misinterpreted as a new medical condition and additional drug therapy is then prescribed to treat this medical condition. (5) Use of multiple medications can lead to problems with medication adherence, compounded by visual or cognitive compromise in many older adults.

Drug therapy is an important component of care in the elderly. Drug prescription is a complex process and includes: deciding that a drug is indicated, choosing the best drug, determining a dose and schedule appropriate for the patient's physiologic status, monitoring for effectiveness and toxicity, educating the patient about expected side effects, and indications for seeking consultation [5]. Several times, polypharmacy results into a problem especially when many drugs are given to patients by multiple healthcare providers working independently of each other. Inappropriate drug prescription results into avoidable adverse drug reaction. Drug prescription in the elderly is a serious challenge and, therefore, the possibility of adverse drug reaction should always be considered when prescribing for older adults. Premarketing drug trials often exclude geriatric patients and the approved doses in the trials may not be appropriate for older adults [6]. Many medications need to be used with special caution because of age-related changes in pharmacokinetics (i.e., absorption, distribution, metabolism, and excretion) and pharmacodynamics (the physiologic effects of the drug) [5]. A number of factors in older individuals contribute to their increased risk for developing a drugrelated problem. These include frailty, coexisting medical problems, memory issues, and use of multiple prescribed and non-prescribed medications [7]. Determine drug dosages when prescribing for older adults requires a lot of care and caution. This is because an increased volume of distribution may result from the proportional increase in body fat relative to skeletal muscle with aging [5]. Decreased drug clearance may result from the natural decline in renal function with age, even in the absence of renal disease [8]. Older patients are frequently exposed to potential drug interactions. A study found out those two-thirds of patients who presented in a teaching hospital had been exposed to at least one potential drug interaction and about $10 \%$ of interactions were considered to be of major clinical significance [9]. The factors that can lead to polypharmacy are both from the patient and physician. Physician factors that play a role in polypharmacy include: presuming that patients expect prescription medications; prescribing drugs without sufficiently investigating clinical situations; providing unclear, complex, or incomplete instructions on how to take a medicine; not simplifying medication regimens as much as possible; not conducting a medication review with the patient on a regular basis; ordering automatic refills without adequate follow-up; and a lack of knowledge of geriatric clinical pharmacology can lead to inappropriate prescribing. The Patient Factors include: Seeing multiple physicians and using multiple pharmacies. This leads to incomplete knowledge by physicians of all the drugs a patient is taking; Hoarding of medications and insisting on taking medications that may no longer be appropriate; Patients not accurately reporting all medicines that they are concurrently taking, or symptoms they are experiencing (that might be drug induced); this can result in duplicate prescriptions or treating drug effects with additional drugs; Patients assuming that once most medications are started, they should be continued indefinitely; Changes in patient's daily habits may affect the action of medications, such as smoking, changes in activity level, food and fluid intake.

Polypharmacy leads to the following problems: (1) More adverse drug reactions, mostly due to over-the-counter medications. The most consistent risk factor for adverse drug reactions is the number of drugs being taken and the risk rises exponentially as the number of drugs increases. (2). Drug-drug interactions. (3). Decreased medication compliance. 4. Poor quality of life, and 5.Unnecessary drug expense Studies have evaluated the unnecessary drug use definition of polypharmacy [10,11]. A study of 236 ambulatory patients aged $>65$ years by Lipton et al. [10] found that almost $60 \%$ of patients were taking medications that were suboptimal or lacking an indication. Schmader et al. [12] had similar findings: they reported that $55 \%$ of 208 elderly patients were taking drugs without an indication. They also found

*Corresponding author: Abdulraheem, Associate Professor, University of Ilorin, Nigeria; E-mail: ibroraheem@yahoo.com

Received August 22, 2013; Accepted August 25, 2013; Published August 26, 2013

Citation: Abdulraheem IS (2013) Polypharmacy: ARisk Factor for Geriatric Syndrome, Morbidity \& Mortality. Aging Sci 1: e103. doi: 10.4172/2329-8847.1000e103

Copyright: ( 2013 Abdulraheem. This is an open-access article distributed unde the terms of the Creative Commons Attribution License, which permits unrestricted use, distribution, and reproduction in any medium, provided the original author and source are credited. 
that one third of patients were taking ineffective drugs, and $16 \%$ had a therapeutic duplication in their medication regimens. In a study of 834 outpatients aged_>65 years, Schmader et al. [13] evaluated unnecessary drug use, defined by the Medication Appropriateness Index (MAI) criteria as a medication with no indication, lack of effectiveness, or therapeutic duplication. The daily mean number of unnecessary drugs in this study was 0.65 per person. A study in frail elderly veterans $(\mathrm{N}=384)$, which also used the MAI to define unnecessary drug use, found that $44 \%$ of patients had more than 1 unnecessary medication at hospital discharge, with $25 \%$ of the patients having the medication started during the hospitalization [14]. The reasons for the unnecessary drug use included no indication (32\%), lack of effectiveness (18\%), and therapeutic duplication (7\%). Gastrointestinal, central nervous system, and therapeutic nutrient/mineral agents were found to be the most commonly used unnecessary drugs. Many risk factors for polypharmacy have been identified and can be classified into 1 of 3 groups: demographic, health status, and access to health care characteristics. Increased aged, white race and education are demographic characteristics associated with polypharmacy [15]. Poorer health, depression, hypertension, anemia, asthma, angina diverticulitis, osteoarthritis, gout, diabetes mellitus, and use of more than 9 medications are the health status characteristic associated with polypharmacy [14]. Predictors of polypharmacy related to access to health care characteristics include number of health care visits, supplemental insurance and multiple providers [16]. A study by Larson et al. [17] showed an increased risk of cognitive impairment with multiple medications. A study by Ruby [18] found that the use of multiple medications with urologic activity increased the risk of urinary incontinence. A study has also examined the impacts of multiple medication use on falls. Those patients taking more than 2 psychotropic agents had a 2.4 to 4.5 folds increased risk of falling than those taking one central nervous system drug [19].

Mininimising or controlling polypharmacy requires periodic evaluation of patient's drug regimen. Periodic evaluation of a patient's drug regimen is an essential component of medical care for an older person [5]. The evaluation may reveal the need to change the prescribed drug therapy. These changes may include discontinuing a therapy prescribed for an indication that no longer exists, substituting a therapy with a potentially safer agent, changing drug dosage, or adding a new medication. A medication review should consider whether a change in patient status (e.g. renal or liver function) might necessitate dosing adjustment, the potential for drug-drug interaction, whether patient symptoms might reflect a drug side effect, or whether the regimen could be simplified [20]. Medication reviews should be done in a systematic manner with the patient meeting the pharmacist within a few weeks of starting a new medication. Patients should be advised to bring to the clinic all medications they are currently using as they may not consider over-the-counter products, ointments, vitamins, ophthalmic preparations, or herbal medicines as drug therapies. Un-necessary therapy should be discontinued. The decision to discontinue medication is determined in part by the goals of care for that patient and the risks of adverse effects for that patient. There are limited studies on how best to withdraw medications [21]. It is important to gradually taper off most medications to minimize withdrawal reactions and to allow symptom monitoring, unless dangerous signs or symptoms indicate a need for abrupt medication withdrawal [5]. Drugs that require tapering, includes beta blockers, opioids, barbiturates, clonidine, gabapentin, and antidepressants. Non-pharmacologic approaches should always be considered in controlling polyphamacy. For example some conditions in older adults may be amenable to lifestyle modification in lieu of pharmacotherapy. The Trial of Non-pharmacologic Interventions in the Elderly (TONE) demonstrated that weight loss and reduced sodium intake could allow discontinuation of antihypertensive medication in about 40 percent of the intervention group [22,23]. Use of safer drug alternatives is also important in the control of polyphamacy. When drug therapy is indicated for the older patient, it may be possible to substitute a safer alternative for the current regimen. For example, in the treatment of non-inflammatory arthritis, acetaminophen may provide adequate pain relief and be a safer alternative to Non- Steroidal Anti-Inflammatory Drugs [24]. The possibility of an adverse drug event (ADE) should always be borne in mind when prescribing for an older adult; any new symptom should be considered drug-related until proven otherwise. Pharmacokinetic changes lead to increased plasma drug concentrations and pharmacodynamic changes lead to increased drug sensitivity in older adults. Clinicians must be alert to the use of herbal and dietary supplements by older patients, who may not volunteer this information and are prone to drug-drug interactions related to these supplements. The role of the pharmacists in the reduction of polypharmacy in the elderly cannot be over emphasized. Pharmacists should evaluate the aspects concerning the use of adequate medications; reduction of medication doses without affecting treatment efficiency; adjustment of doses beyond the drug safety margin; and correct use of the medication by elderly patients [25]. Adherence to medication by the elderly patient is another factor that contributes to polypharmacy reduction. Medication adherence can be defined as consistency between medical prescription and drugs consumed by the patient [26] Another factor that could contribute to reduce polypharmacy complications is the reduction on conflicting information given by many different health professionals [27].

\section{References}

1. Ferner RE, Aronson JK (2006) Communicating information about drug safety BMJ 333:143.

2. Tinetti ME, Bogardus ST Jr, Agostini JV (2004) Potential pitfalls of diseasespecific guidelines for patients with multiple conditions. N Engl J Med 351: 2870-2874.

3. Lai SW, Liao KF, Liao CC, Muo CH, Liu CS, et al. (2010) Polypharmacy correlates with increased risk for hip fracture in the elderly: a population-based study. Medicine (Baltimore) 89: 295-299.

4. Rochon PA, Gurwitz JH (1997) Optimising drug treatment for elderly people: the prescribing cascade. BMJ 315: 1096-1099.

5. Paul A Rochon. Drug prescribing for older adults.

6. Cho S, Lau SW, Tandon V, Kumi K, Pfuma E, et al. (2011) Geriatric drug evaluation: where are we now and where should we be in the future?. Arch Intern Med 171: 937-940.

7. Mallet L, Spinewine A, Huang A (2007) The challenge of managing drug interactions in elderly people. Lancet 370:185-191.

8. Rowe JW, Andres R, Tobin JD, Norris AH, Shock NW (1976) The effect of age on creatinine clearance in men: a cross-sectional and longitudinal study. J Gerontol 31:155-163.

9. Mukasa D, Hughes J (2002) Drug interactions in the elderly: frequency significance and differences among standard reference resources. Aust Pharmacist 21: 603-608.

10. Lipton HL, Bero LA, Bird JA, McPhee SJ (1992) The impact of clinical pharmacists consultations on physicians geriatric drug prescribing. A randomized control trial. Med Care 30: 646-658.

11. Steimam MA, Landefeld CS, Rosenthat GE, Berthenthal D, Sen S, et al. (2006) Polypharmacy and prescribing quality in older people JAm Geriatr Soc 54: 1516-1523.

12. Scmalder K, Hanlon JT, Weinbergam, Landsman PB, Samsa GP, et al. (1994) Appropriateness of Medication prescribing in ambulatory elderly patients. J Am Geriatr Sov 42: 1241-1247.

13. Schmander KF, Hanlon JT, Peiper CF, Sloane R, Ruby CM (2004) Effects 
Citation: Abdulraheem IS (2013) Polypharmacy: A Risk Factor for Geriatric Syndrome, Morbidity \& Mortality. Aging Sci 1: e103. doi: 10.4172/2329$8847.1000 \mathrm{e} 103$

of geriatric evaluation and management on adverse drug reaction and suboptimal prescribing in the frail elderly. Am J Med 116: 394-401.

14. Hajjar ER, Hanlon JT, Sloane RT, Lindblad CI, Pieper CF, et al. (2005) Unnecessary drug in frail older people at hospital discharge. JAm Geriatry Soc 53: 1518-1523.

15. Linijakumpu T, Hartikainen S, Klankka T, Veijola J, Kivela SL, et al. (2002) Use of medications \& polypharmacy are increasing among the elderly. $\mathrm{J}$ Clin Epidelol 55: 809-817.

16. Jorgensen T,Johansson S, Kennerfalk A, Wallander MA, Svardsudd K (2001) Prescription drug use, diagnosis and health care utilization among the elderly. Ann Pharmacotiser 35:1004-1009.

17. Larson EB, Kukull WA, Buchner D, Reitler BR (1987) Adverse drug reactions associated with global cognitive impairment in elderly persons. Ann Intern Med 107: 169-173.

18. Ruby CM, Hanlon JJ, Fillenbaum GG, Pieper CF, Branch LG, et al. Medication use and control of urination among community dwelling older aults. J Ageing Health 17: 661-674.

19. Fink H, Wyman J, Hanton JT (2005) Brackdursti text book of Geriatric medicine \& Gerontology.

20. George CJ, Jacobs LG (2011) Geriatrics medication management rounds: a novel approach to teaching rational prescribing with the use of the medication screening questionnaire. J Am Geriatr Soc 59:138-142.
21. Steinman MA, Hanlon JT (2010) Managing medications in clinically complex elders: "There's got to be a happy medium". JAMA 304:1592-1601.

22. Appel LJ, Espeland MA, Easter L, Wilson AC, Folmar S, et al. Effects of reduced sodium intake on hypertension control in older individuals: results from the Trial of Nonpharmacologic Interventions in the Elderly (TONE). Arch Intern Med 161: 685-693.

23. Whelton PK, Appel LJ, Espeland MA, Applegate WB, Ettinger WH Jr, et al (1998) Sodium reduction and weight loss in the treatment of hypertension in older persons: a randomized controlled trial of nonpharmacologic interventions in the elderly (TONE). TONE Collaborative Research Group. JAMA 279: 839846.

24. Bradley JD, Brandt KD, Katz BP, Kalasinski LA, Ryan SI (1991) Comparison of an antiinflammatory dose of ibuprofen, an analgesic dose of ibuprofen, and acetaminophen in the treatment of patients with osteoarthritis of the knee. $\mathrm{N}$ Engl J Med 325: 87-91.

25. Simonson W, Feinberg JL (2005) Medication-related problems in the elderly: defining the issues and identifying solutions. Drugs Aging 22: 559-569.

26. Murray MD, Morrow DG, Weiner M, Clark DO, Tu W, et al. (2004) A conceptual framework to study medication adherence in older adults. Am J Geriatr Pharmacother 2: 36-43.

27. Field TS, Mazor KM, Brisacher B, Debellis KR, Gurwitz JH (2007) Adverse drug events resulting from patient errors in older adults. J Am Geriatr Soc 55: 271 276 . 